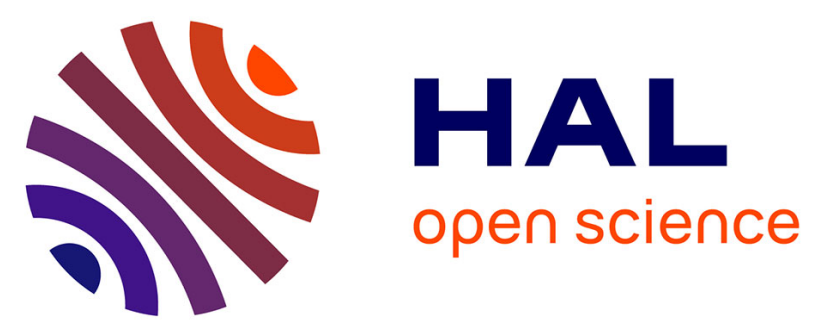

\title{
Performance improvement of small-scale rotors by passive blade twist control
}

Peng Lv, Sebastien Prothin, Fazila Mohd Zawawi, Emmanuel Bénard, Joseph Morlier, Jean-Marc Moschetta

\section{- To cite this version:}

Peng Lv, Sebastien Prothin, Fazila Mohd Zawawi, Emmanuel Bénard, Joseph Morlier, et al.. Performance improvement of small-scale rotors by passive blade twist control. Journal of Fluids and Structures, 2015, 55, pp.25-41. 10.1016/j.jfluidstructs.2015.01.008 . hal-01847334

\section{HAL Id: hal-01847334 https://hal.science/hal-01847334}

Submitted on 23 Jul 2018

HAL is a multi-disciplinary open access archive for the deposit and dissemination of scientific research documents, whether they are published or not. The documents may come from teaching and research institutions in France or abroad, or from public or private research centers.
L'archive ouverte pluridisciplinaire HAL, est destinée au dépôt et à la diffusion de documents scientifiques de niveau recherche, publiés ou non, émanant des établissements d'enseignement et de recherche français ou étrangers, des laboratoires publics ou privés. 


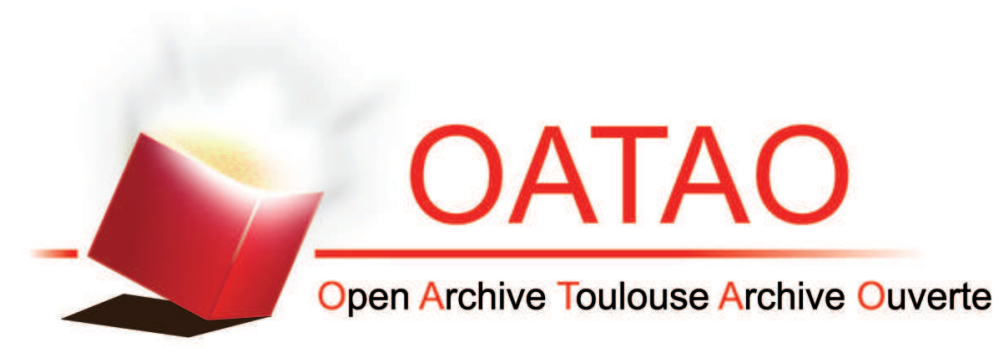

\section{Open Archive Toulouse Archive Ouverte (OATAO)}

OATAO is an open access repository that collects the work of some Toulouse researchers and makes it freely available over the web where possible.

This is an author's version published in: https://oatao.univ-toulouse.fr/18615

Official URL:https://doi.org/10.1016/j.jluidstructs.2015.01.008

\section{To cite this version :}

Lv, Peng and Prothin, Sebastien and Mohd Zawawi, Fazila and Bénard, Emmanuel and Morlier, Joseph and Moschetta, Jean-Marc Performance improvement of small-scale rotors by passive blade twist control. (2015) Journal of Fluids and Structures, vol. 55. pp. 25-41. ISSN 0889-9746

Any correspondence concerning this service should be sent to the repository administrator: tech-oatao@listes-diff.inp-toulouse.fr 


\title{
Performance improvement of small-scale rotors by passive blade twist control
}

\author{
Peng Lv ${ }^{a}$, Sebastien Prothin ${ }^{a}$, Fazila Mohd-Zawawi ${ }^{a}$, Emmanuel Benard ${ }^{a}$, \\ Joseph Morlier ${ }^{\mathrm{b}}$, Jean-Marc Moschetta ${ }^{\mathrm{a}}$ \\ a Département Aérodynamique, Energétique et Propulsion (DAEP), Institut Supérieur de l'Aéronautique et de l'Espace, \\ Toulouse 31055, France \\ bépartement Mécanique des Structures et Matériaux (DMSM), Institut Supérieur de l'Aéronautique et de l'Espace, Toulouse 31055, France
}

Keywords:

Small-scale

Flexible blade

Centrifugal force

Tip mass

Laser Displacement Sensor

\begin{abstract}
A B S T R A C T
A passive twist control is proposed as an adaptive way to maximize the overall efficiency of the small-scale rotor blade for multifunctional aircrafts. Incorporated into a database of airfoil characteristics, Blade Element Momentum Theory is implemented to obtain the blade optimum twist rates for hover and forward flight. In order to realize the required torsion of blade between hover and forward flight, glass/epoxy laminate blade is proposed based on Centrifugal Force Induced Twist concept. Tip mass is used to improve the nosedown torsion and the stabilization of rotating flexible blade. The laminate blades are tested in hover and forward flight modes, with deformations measured by Laser Displacement Sensor. Two Laser Displacement Sensors are driven by the tracking systems to scan the rotating blade from root to tip. The distance from blade surface to a reference plane can be recorded section by section. Then, a polynomial surface fitting is applied to reconstruct the shape of rotating blade, including the analysis of measurement precision based on the Kline-McClintock method. The results from deformation testings show that nose-down torsion is generated in each flight mode. The data from a Fluid Structure Interaction model agrees well with experimental results at an acceptable level in terms of the trend predictions.
\end{abstract}

\section{Introduction}

Multifunctional aircrafts can fly in hover like a helicopter and forward flight like an airplane. Designing a rotor to operate efficiently in hover and forward flight presents a challenge since the inflow velocity and thrust requirement for each flight condition are quite distinct. In hover, the inflow velocity is small and the rotor must provide high thrust to support the aircraft weight. By contrast, in forward flight, the inflow velocity is relatively large and the low thrust must only overcome the drag. The large difference in inflow velocity and the thrust requirement for each flight condition suggests varied blade shapes, such as chord and twist.

The blade twist of XV-15 rotor aircraft was obtained through linear interpolation of twist between ideal rotor and propeller by a compromise analysis (McVeigh and Rosenstein, 1983). Although this trade-off solution provided an acceptable performance on XV-15, the stiff rotor with certain twist cannot maximize the overall efficiency. In the early 1980s, the study of passive blade twist control was started to improve the performance of rotor. The extension-twist coupling blade was applied on XV-15 rotor in two designs (Bauchau et al., 1983). This approach provided torsion for the improvement of rotor 
efficiency. The investigation was conducted to observe if the required twist deformation of full-scale extension-twist coupling rotor blades can be achieved within material design limit loads (Nixon, 1987). The results showed that the design is sufficient to satisfy the torsion requirements at design limit loads. A passive blade twist control method was proposed for the rotor on XV-15 (Nixon, 1988). If the optimum linear twists for hover and forward flight, instead of the compromise linear twist can be achieved, the rotor efficiency can be improved evidently. The study demonstrated successfully the feasibility of the passive blade control on conventional rotor aircraft. The feasibility of passive twist control for composite rotor blades was studied further using additional experiments (Lake et al., 1994). Since a typical tiltrotor blade would require much higher twist rate changes, the authors finally suggested the addition of tip masses to reach high twist rates for practical applications. The unique aeroelastic tailoring concepts of composite materials were discussed for aeroelastic stability and aerodynamic performance of rotor aircrafts (Nixon et al., 2000), e.g. bending-twist coupling and extension-twist coupling. The small-scale rotors on Micro Air Vehicles (MAVs) also suffer the problem caused by different blade twist rates between hover and forward flight (Shkarayev et al., 2008). Due to the small size of rotors on MAVs, the complex tailored cross section of blade for passive twist control based on conventional tiltrotor aircraft is not available any more. A small-scale flexible and stowable rotor was applied on a micro helicopter (Sicard, 2011). However, the blade was proposed to reduce impact damage and access confined spaces instead of to increase the aerodynamic efficiency of rotor in terms of torsion generation. Therefore, in current study, a laminate blade is introduced as applied to small-scale rotor.

A key issue to study flexible blade is to measure the deformation of rotating blade. Optical measurement techniques have been developing for some years in aerodynamics, materials and structure, such as Holographic Interferometry (HI), Electronic Speckle Pattern Interferometry (ESPI), Projection Moiré Interferometry (PMI) and Digital Image Correlation (DIC) (Rajpal, 2009; Schmidt and Tyson, 2003). There are limited investigations of optical measurement techniques for rotor blade deformation. The PMI technique was presented to obtain quantitative deformation profiles conditionally sampled as a function of rotor azimuth (Fleming and Gorton, 1998). Experimental results showed blade bending, twist, and unsteady motion. This initial proof-of-concept test was demonstrated the capability of PMI to acquire accurate, full field data of blade deformation. PMI was then used in wind tunnel tests to obtain azimuthally dependent blade bending and twist for a 4bladed Active Twist Rotor (ATR) (Fleming et al., 2002). The measurement helps to understand the overall behavior of the ATR system and the physical mechanisms causing the reduction in rotor loads and noise. Blade deflection measurements using stereo photogrammetry were performed for a UH-60A 4-bladed rotor system (Olson et al., 2010). The ability to photogrammetrically measure blade deflection during wind tunnel testing was successfully demonstrated. The deformation of small-scale rotating blade was measured using DIC system (Lawson and Sirohi, 2011). A commercial DIC software was used to obtain bending and twist on three different types of rotors with varying flexibility. As discussed above, PMI was found to have low sensitivity for in-plane deformation and moderate for out-of-plane deformation. By contrast, DIC has a relatively high sensitivity that can reach $1 / 30000$ of the test field. However, it needs a pre-processing which is to apply a stochastic speckle pattern to the surface by spraying it with a high-contrast and non-reflective paint. The painting in preprocessing will probably affect the stiffness of small-scale blades.

In this study, Blade Element Momentum Theory (BEMT) model was implemented to obtain the optimum twist rates of blade in hover and forward flight. A concept of Centrifugal Force Induced Twist (CFIT) was proposed using glass/epoxy and tip mass to generate stable torsion of rotating blade. A Laser Displacement Sensor (LDS) rig and corresponding postprocessing method were developed to measure the deformation of rotating blade. The experimental results were compared with Fluid Structure Interaction (FSI) model in terms of bending and torsion deformations.

\section{Optimum blade twist rates in each flight mode}

Incorporated into a database of airfoil characteristics, a model based on Blade Element Momentum Theory is implemented to obtain the optimum blade twist rates for hover and forward flight. The BEMT is a method that combines the Blade Element Theory (BET) and Blade Momentum Theory (BMT) in order to estimate the inflow distribution (Leishman, 2006; Adkins, 1990). In the classical approach of rotor analysis, lift polar is a linear function. To solve the BEMT equations, the blade should be numerically discretized into a series of small elements. In rotor mode, solution of induced flow ratio $\lambda$ can be solved by Eq. (1) through a few iterations:

$$
4 F \lambda^{2} r d r=\frac{1}{2} \sigma C_{l} r^{2} d r
$$

where $\lambda$ is the inflow ratio, $F$ is the Prandtl's tip-loss factor, $r$ is the non-dimensional radius, $d r$ is the non-dimensional length of each element, $C_{1}$ is the local lift coefficient and $\sigma$ is the local solidity ratio. The BEMT model used for propeller mode includes the swirl induced velocity. The analysis procedure requires an iterative solution for the inflow angle at each radial position. The inflow angle $\phi$ is defined by

$$
\tan \phi=\frac{V_{c}(1+a)}{\Omega r_{1}\left(1-a^{\prime}\right)}
$$

where $V_{\mathrm{c}}$ is the inflow velocity, $a$ and $a^{\prime}$ are the interference factors respectively in the axial and swirl directions, $\Omega$ is the rotational speed and $r_{1}$ is the local radius. 
Figure of Merit (FM) is used to compare the hovering efficiency of different rotors at the same disk loading. BEMT results can provide the total thrust coefficient $C_{\mathrm{T}}$ and the total power coefficient $C_{\mathrm{P}}$ by

$$
\begin{aligned}
& C_{\mathrm{T}}=\frac{T}{\rho \pi R^{2}(\Omega R)^{2}}, \\
& C_{\mathrm{P}}=\frac{P}{\rho \pi R^{2}(\Omega R)^{3}},
\end{aligned}
$$

where $T$ is the thrust, $P$ is the power, $\rho$ is the density of the fluid and $R$ is the blade radius. The FM in hover can be written as

$$
\mathrm{FM}=\frac{C_{\mathrm{T}}{ }^{3 / 2} / \sqrt{2}}{C_{\mathrm{P}}} .
$$

By contrast, the propulsive efficiency of blade in forward flight can be expressed by

$$
\eta=\frac{\lambda_{\infty} C_{\mathrm{T}}}{C_{\mathrm{P}}}=\frac{J C_{\mathrm{T}}}{\pi C_{\mathrm{P}}},
$$

where $\lambda_{\infty}$ is the tip speed ratio $\left(\lambda_{\infty}=V_{\mathrm{c}} / \Omega R\right)$ and $J$ is the advance ratio $\left(J=V_{\mathrm{c}} / N_{\mathrm{s}} D\right)$. $N_{\mathrm{s}}$ is the revolution per second and $D$ is the blade diameter. The values of tip speed ratio $\lambda_{\infty}$ and advance ratio $J$ are related by $\lambda_{\infty}=J / \pi$. The database of airfoil characteristics were obtained from XFOIL which is an airfoil design and analysis code based on vortex panel method (Drela, 1989). BEMT model was validated using existing performance data of rotor and propeller (Bohorquez, 2007; Smedresman et al., 2011).

MAVion, a tilt-body MAV developed by ISAE, is designed to fly in both hover and forward flight. Table 1 exhibits the operation conditions of MAVion for both hover and forward flight. In order to obtain the optimum twist rates for hover and forward flight for a given thrust requirement, a matrix of blades with same chord and varied linear twist are analyzed and compared using the BEMT model. The blade geometry parameters are given in Table 2. The Reynolds number based on the uniform chord and velocity at $0.75 R$ is below 100000 . Thrust coefficient is adjusted by collective pitch for the five blades to reach the thrust requirements.As it can be seen in Fig. 1(a), Figure of Merit (FM) increases with reducing blade twist in rotor mode. Low twist rate $-10^{\circ}$ helps to improve the FM by $23 \%$. Fig. 1(b) demonstrates that propulsive efficiency increases with increasing blade twist in propeller mode. High twist rate $-30^{\circ}$ improves the propulsive efficiency by $17 \%$. Hence, a flexible blade can improve the overall efficiency of rotor if it can realize the twist deformation for different flight modes. Finally, according to the mission requirement of MAVion, the solution of proprotor for MAVion is given in Table 3.

\section{Exploration of the flexible blade}

\subsection{Concept of Centrifugal Force Induced Twist}

A deformable, durable and stable blade for small-scale rotor is characterized by the deformation, failure performance and dynamics behavior. To be specific, it is capable of deforming in terms of twist to adapt different flight conditions. Meanwhile, the deformation should be stable during the rotation. Moreover, it should be practical to be applied on MAVs without size problem. Besides, it can take the airloads without large blade bending. Finally, this adaptive rotor should have an acceptable fatigue performance. The initial step of the procedure is to select suitable reinforcing fiber for the laminate blade. The significant factor to select a reinforcing fiber for small-scale rotor is linked to its tailoring capacity. Glass/epoxy was

Table 1

Operation conditions of MAVion.

\begin{tabular}{llc}
\hline & Hover & Forward flight \\
\hline RPM & 1500 & 1200 \\
Inflow velocity (m/s) & 0 & 10 \\
Advance ratio & - & 1.25 \\
Thrust (N) & 2 & 0.3 \\
Thrust coefficients & 0.01 & 0.003 \\
\hline
\end{tabular}

Table 2

Blade geometry parameters.

\begin{tabular}{ll}
\hline Hub radius $(\mathrm{mm})$ & 15 \\
Radius $(\mathrm{mm})$ & 200 \\
Chord $(\mathrm{mm})$ & 30 \\
Number of blades & 2 \\
Airfoil & Flat plate $(2.5 \%$ thickness $)$ \\
\hline
\end{tabular}


(a)

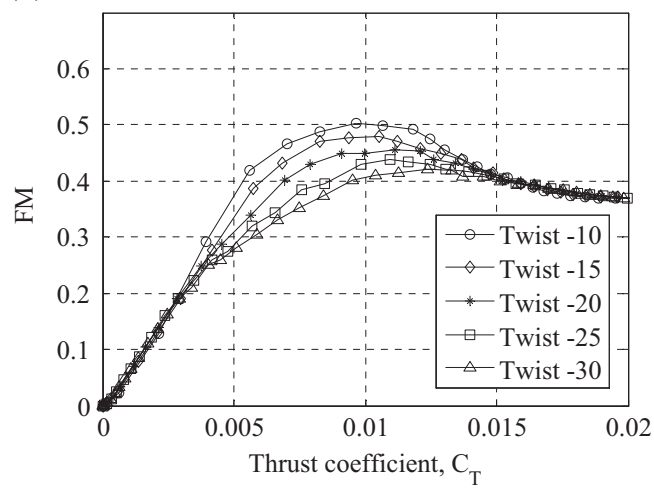

(b)

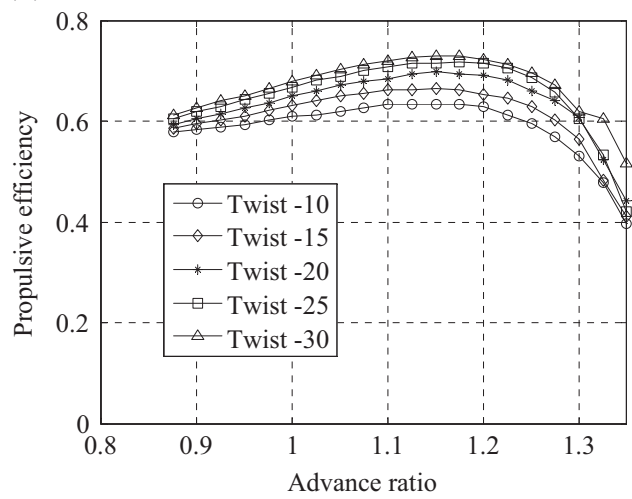

Fig. 1. Blade twist effects on aerodynamic efficiency: (a) rotor mode, $C_{\mathrm{T}}=0.01$ and (b) propeller mode, $C_{\mathrm{T}}=0.003$.

Table 3

Optimized proprotor of MAVion.

\begin{tabular}{lrl}
\hline & Hover & Forward flight \\
\hline Twist (deg) & -10 & \multicolumn{1}{c}{-30} \\
Collective pitch (deg) & 31 & 54 \\
\hline
\end{tabular}

determined for the flexible blade of small-scale rotor in current study. In order to improve the aerodynamic performance of flat plate at low Reynolds number, the thickness of laminate blade should be thin enough to provide a low thickness-tochord ratio (Hein and Chopra, 2005). Thus, two typical laminate configurations are considered here, a symmetric laminate

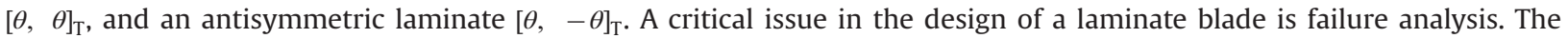
comparison of strength of Uni-Directional (UD) material and angle-ply laminates was described based on a typical carbon/ epoxy composite (AS4/3501-6) using first ply failure analysis (Daniel and Ishai, 1994). All of the uniaxial tensile strength, uniaxial compressive strength and in-plane shear strength of the angle-ply laminate are evidently higher than those of the off-axis UD material. Thus, in current study, Antisymmetric laminate $[45,-45]_{\mathrm{T}}$. was employed as balanced laminates (Fig. 2). The elastic constants of glass/epoxy are given in Table 4, which are longitudinal Young's modulus $E_{1}$, transverse Young's modulus $E_{2}$, Poisson's ratio $v_{12}$ and shear modulus $G_{12}$.

A concept of Centrifugal Force Induced Twist (CFIT) was proposed for the adaptive rotor. In CFIT flexible concept, tip mass is designed to provide the nose-down twisting moment and produce the torsion of laminate blades. Fig. 3 demonstrates the tip mass which can slide in the chordwise direction at blade tip. The concept of CFIT for adaptive rotor is described in Fig. 4. The blade is rotating in the anti-clockwise direction at a rotational speed $\Omega$. A position factor $k$ is used to define the position of tip rod in the chordwise direction at blade tip. The length of tip rod is represented by $L$. There is a mass element $d m$ on the rotating blade which can generate a certain centrifugal force $F_{\mathrm{C}}$. It has a component in the chordwise direction of blade. The component tends to flat the blade cross section with an initial pitch angle $\theta$. This is the method of CFIT blade generating torsion. 


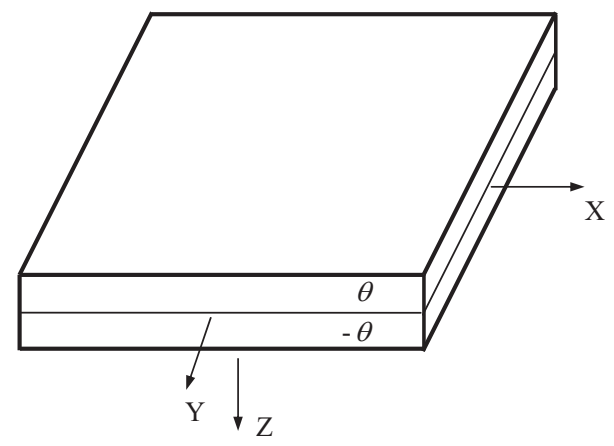

Fig. 2. Schematic illustration of a laminate with two antisymmetric layers.

\section{Table 4}

Elastic constants of E-glass/epoxy.

\begin{tabular}{llll}
\hline$E_{1}(\mathrm{GPa})$ & $E_{2}(\mathrm{GPa})$ & $v_{12}$ & $G_{12}(\mathrm{GPa})$ \\
\hline 38.0 & 10.0 & 0.25 & 4.0 \\
\hline
\end{tabular}

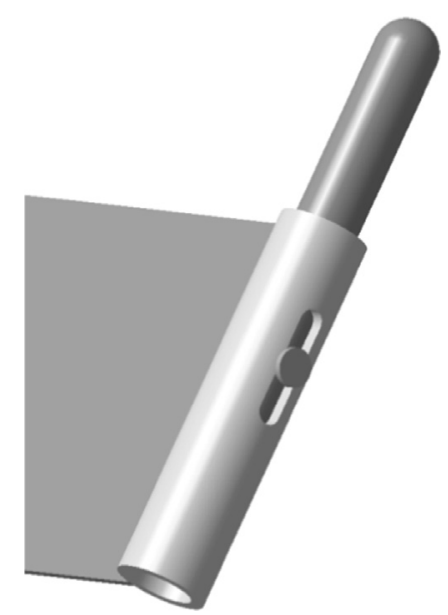

Fig. 3. Close-up of tip mass model.

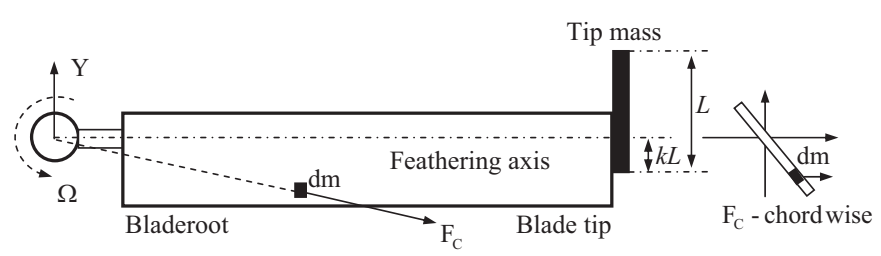

Fig. 4. Schematic of Centrifugal Force Induced Twist.

At any local section of blade, the total centrifugal force and total nose-down moment are defined (Sicard, 2011):

$$
\begin{aligned}
& F_{\mathrm{C}}(x)=\int_{x}^{R} \Omega^{2} m_{\mathrm{x}} d x+m_{\mathrm{T}} \Omega^{2} R, \\
& M_{\mathrm{ND}}(x)=I_{\theta} \Omega^{2}(R-x) \sin \theta+I_{\mathrm{T}} \Omega^{2} \sin \theta_{\mathrm{T}},
\end{aligned}
$$

where $R$ is the rotor radius, $\Omega$ is the rotation speed, $m_{\mathrm{x}}$ is the local mass of laminate blade and $m_{\mathrm{T}}$ is the tip mass. $x$ corresponds the spanwise coordinate while $y$ represents the chordwise coordinate. In addition, $\theta$ is the local twist angle 
and $\theta_{\mathrm{T}}$ represents the twist angle at the blade tip. $I_{\theta}$ is the torsional moment of inertia of the blade and $I_{\mathrm{T}}$ is the torsional moment of inertia of the tip rod. The torsional moment of inertia of the blade $I_{\theta}$ can be expressed by

$$
I_{\theta}=\int_{0.15 R}^{R} \int_{-0.75 C}^{0.25 C} \rho_{\mathrm{B}} y^{2} t_{\mathrm{B}} d x d y,
$$

where $C$ is the chord of blade, $\rho_{\mathrm{B}}$ is the density of it and $t_{\mathrm{B}}$ is blade thickness. The torsional moment of inertia of the tip rod $I_{\mathrm{T}}$ is defined as

$$
I_{\mathrm{T}}=\int_{-k L}^{(1-k) L} \rho_{\mathrm{T}} y^{2} s d y,
$$

where $\rho_{\mathrm{T}}$ is the density of tip rod and $s$ is the cross section area. For a given blade with a tip mass rotating at certain RPM, the nose-down pitching moment can be determined by Eq. (8). This nose-down pitching moment will be used to estimate the blade torsion generated during rotation.

\subsection{Torsion estimation}

The Classical Lamination Theory (CLT) demonstrates that, for a general composite laminate, the forces and moments on it are related to the strains and curvatures at reference surface:

$$
\left[\begin{array}{c}
N_{\mathrm{x}} \\
N_{\mathrm{y}} \\
N_{\mathrm{xy}} \\
M_{\mathrm{x}} \\
M_{\mathrm{y}} \\
M_{\mathrm{xy}}
\end{array}\right]=\left[\begin{array}{cccccc}
A_{11} & A_{12} & A_{16} & B_{11} & B_{12} & B_{16} \\
A_{12} & A_{22} & A_{26} & B_{12} & B_{22} & B_{26} \\
A_{16} & A_{26} & A_{66} & B_{16} & B_{26} & B_{66} \\
B_{11} & B_{11} & B_{11} & D_{11} & D_{11} & D_{16} \\
B_{12} & B_{22} & B_{26} & D_{12} & D_{22} & D_{26} \\
B_{16} & B_{26} & B_{66} & D_{16} & D_{26} & D_{66}
\end{array}\right]\left[\begin{array}{c}
\varepsilon_{\mathrm{x}}^{0} \\
\varepsilon_{\mathrm{y}}^{0} \\
\varepsilon_{\mathrm{xy}}^{0} \\
\kappa_{\mathrm{x}}^{0} \\
\kappa_{\mathrm{y}}^{0} \\
\kappa_{\mathrm{xy}}^{0}
\end{array}\right],
$$

where the $6 \times 6$ matrix consisting of the components $A_{i j}, B_{i j}$ and $D_{i j}(i, j=1,2,6)$ is laminate stiffness matrix, is also called $A B D$ matrix (Daniel and Ishai, 1994). In order to be able to obtain the strains and curvatures at the reference surface in terms of the force and moment resultants, the inverse of Eq. (11) is as follows:

$$
\left[\begin{array}{c}
\varepsilon_{\mathrm{x}}^{0} \\
\varepsilon_{\mathrm{y}}^{0} \\
\varepsilon_{\mathrm{xy}}^{0} \\
\kappa_{\mathrm{x}}^{0} \\
\kappa_{\mathrm{y}}^{0} \\
\kappa_{\mathrm{xy}}^{0}
\end{array}\right]=\left[\begin{array}{llllll}
a_{11} & a_{12} & a_{16} & b_{11} & b_{12} & b_{16} \\
a_{12} & a_{22} & a_{26} & b_{12} & b_{22} & b_{26} \\
a_{16} & a_{26} & a_{66} & b_{16} & b_{26} & b_{66} \\
b_{11} & b_{11} & b_{11} & d_{11} & d_{11} & d_{16} \\
b_{12} & b_{22} & b_{26} & d_{12} & d_{22} & d_{26} \\
b_{16} & b_{26} & b_{66} & d_{16} & d_{26} & d_{66}
\end{array}\right]\left[\begin{array}{c}
N_{\mathrm{x}} \\
N_{\mathrm{y}} \\
N_{\mathrm{xy}} \\
M_{\mathrm{x}} \\
M_{\mathrm{y}} \\
M_{\mathrm{xy}}
\end{array}\right],
$$

where the $6 \times 6$ matrix consisting of the components $a_{i j}$, $b_{i j}$ and $d_{i j}(i, j=1,2,6)$ is laminate compliance matrix. With respect to torsion, it is given by the curvature:

$$
\kappa_{\mathrm{xy}}^{0}=b_{16} N_{\mathrm{x}}+b_{26} N_{\mathrm{y}}+b_{66} N_{\mathrm{xy}}+d_{16} M_{\mathrm{x}}+d_{26} M_{\mathrm{y}}+d_{66} M_{\mathrm{xy}},
$$

where $N_{\mathrm{x}}, N_{\mathrm{y}}$ and $N_{\mathrm{xy}}$ are the tensile forces in the directions of $X, Y$ and $X Y$. Besides, $M_{\mathrm{x}}, M_{\mathrm{y}}$ and $M_{\mathrm{xy}}$ represent the moments in varied directions. The terms $b_{16}, b_{26}, b_{66}, d_{16}, d_{26}, d_{66}$ are the corresponding compliance factors. They are determined by material properties, thickness and the orientation of the plies of the laminate blade. Since the nose-down pitching moment $M_{\mathrm{ND}}$ is obtained, the torsion of rotating laminate blade can be calculated directly using Eq. (13). Fig. 5 shows the tip twist of rotating laminate blade with the variation of tip mass position at $1500 \mathrm{RPM}$. The torsion of adaptive rotor is generated at the reasonable level which is between $0^{\circ}$ and $-30^{\circ}$.

\subsection{Global center of gravity of blade}

To achieve stable operation of rotating blade, the elastic axis should be ahead of the aerodynamic center and the blade section center of gravity (CG) should be ahead of the elastic axis (Winston, 1968). Pitch-flap flutter and divergence can be avoided if the global CG is ahead of the aerodynamic center (Sicard, 2011). Furthermore, if the tip mass is not suitable to generate a certain nose-down moment and produce an optimum twist, it could cause an unwanted flow-induced vibration. Thus, there should be enough tip weight to adjust the global CG and generate nose-down moment. A large end mass is beneficial for adjusting the global CG and stabilizing the rotating flexible blade, but, it also decreases the weight efficiency of the MAVs. Hence, the compromise weight of tip mass is around $0.0637 \mathrm{~N}$ and in the same level of blade weight $0.0686 \mathrm{~N}$.

According to the equilibrium of moment, the global CG position of laminate blade, expressed as the non-dimensional parameter $\left(y_{\mathrm{CG}}-y_{\mathrm{LE}}\right) / C$, can be expressed as a function of position $k$, as defined in Fig. 4. $y_{\mathrm{CG}}$ and $y_{\mathrm{LE}}$ are the global CG position and the leading edge, respectively. For the considered flat plate airfoil, the aerodynamic center location $\left(y_{\mathrm{AC}}-y_{\mathrm{LE}}\right) / \mathrm{C}$ 


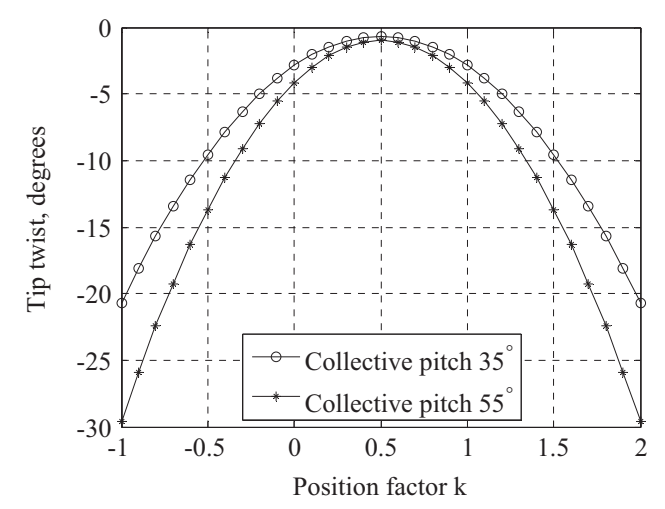

Fig. 5. Torsion prediction.

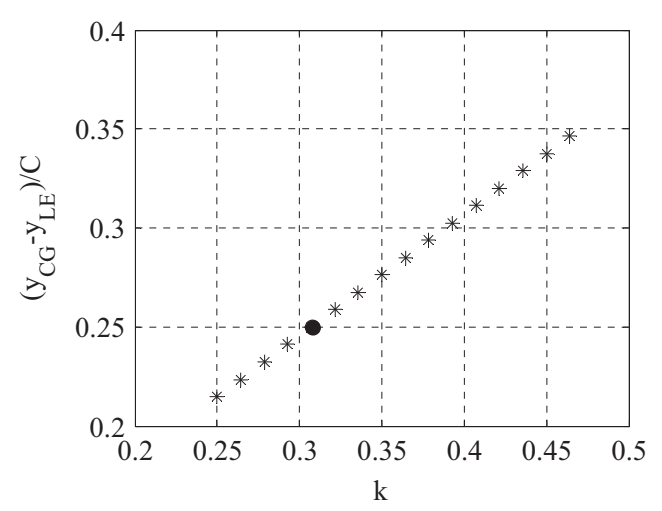

Fig. 6. Global CG.

is approximately equal to 0.25 . When $k$ is decreased (sliding the tip mass forward), the global CG position approaches the position of aerodynamic center. For $k$ values lower than 0.3 , the global CG is ahead of aerodynamic center (Fig. 6). Therefore, the current tip mass can help in improving the stability of rotating blade.

\section{Deformation behavior of rotating flexible blade}

\subsection{Laser displacement sensor rig}

The small-scale rotor with flexible blades is shown in Fig. 7. The bending and torsion distributions during rotation are considered as the blade deformation, since beneficial torsion can increase the overall performance while bending tends to decrease the thrust. In order to measure the deformation of rotating laminate blade, a LDS rig was developed, as shown in Fig. 8. To avoid any disturbance on flow field, the two LDSs are fixed at an incidence angle to measure the blade deformation. They are driven by the tracking systems to scan the rotating blade from root to tip. KEYENCE LK-G502 LDS can record the distance from blade surface to a measuring plane when the rotating blade passes by laser beam. Finally, the data in spanwise direction and chordwise direction are used to reconstruct the shape of rotating blade.

The inflow for measurements in propeller mode was provided by a fan since it is not practical to mount LDS rig inside wind tunnel. The open fan has a diameter of $0.6 \mathrm{~m}$. In order to evaluate the homogeneity and turbulence intensity of the inflow, a Particle Image Velocimetry (PIV) system was used for the downstream station ( $2.5 \mathrm{~m}$ away from the fan outlet). The sampling frequency was $2 \mathrm{~Hz}$ and the number of image pairs was limited to 100. Fig. 9 shows the axial velocity distribution along the radius at varied controlling frequencies. The maximum difference of the axial velocity was found to be $16.3 \%$. Over the range of test configurations, the spatial average value of turbulence intensity was approximately equal to $17.3 \%$. The turbulence intensity is clearly higher than conventional wind tunnels. On one hand, the inflow with high turbulence intensity provided by the fan must cause the unsteady effect of airloads on the rotating blade. On the other hand, the experiments still reveal the essential airload phenomena.

Optical sensor OPTEC OPB608V is a reflective switch which allows the user to observe the pattern shining on a reflective object and provide RPM signal. High torque brushless motor AXI 2808/24 was used in experiments. The voltage range is 


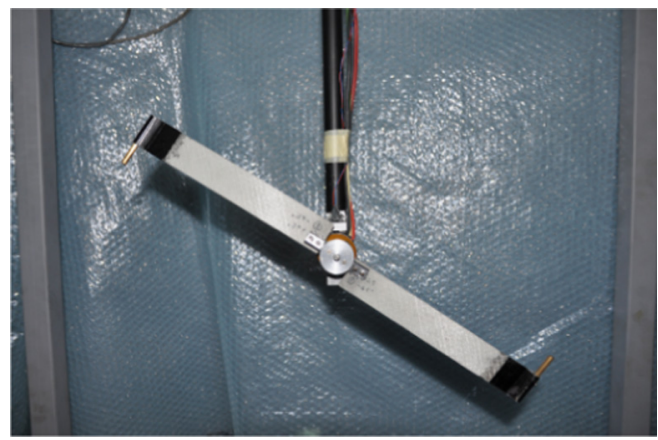

Fig. 7. Close-up of flexible proprotor with tip mass.

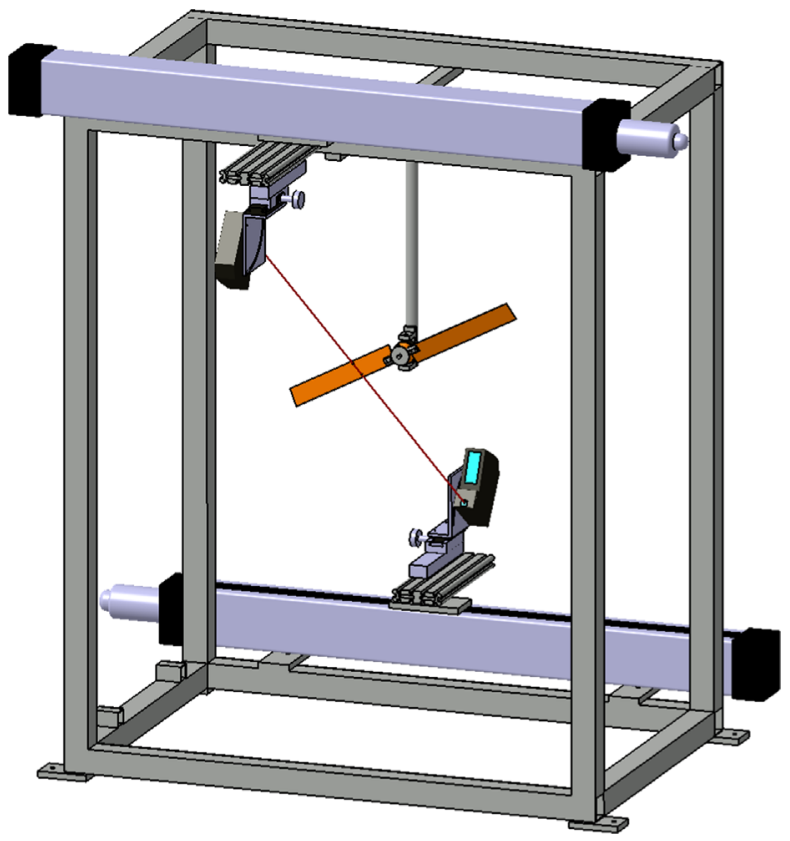

Fig. 8. LDS rig.

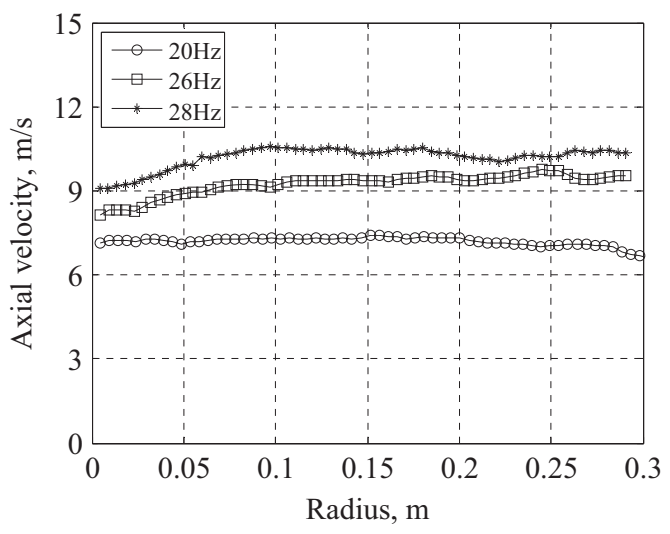

Fig. 9. Axial velocity distribution.

from 4 to 10 cells and the maximum loading of it is $22 \mathrm{~A} / 30 \mathrm{~s}$. The data from test rig was collected by the electronic data acquisition system. The hardware used for signal conditioning and data collection is National Instruments (NI) PCI 6251 board and NI BNC-2090 carrier box. NI PCI 6251 is a high-speed multifunction M series data acquisition (DAQ) board 
optimized for high accuracy at fast samplings. A custom program for the tests was developed based on Labview8.6. There were 10000 samples per second in all tests for each channel.

Based on the mission of MAVion and its optimum proprotor (Tables 1 and 3 ), the collective pitch $35^{\circ}$ was selected for the rotor mode tests while $55^{\circ}$ was used for the experiments in propeller mode. Besides, high collective helps in generating a relatively large torsion in propeller mode. In rotor mode, the blades were tested to explore the RPM effect on deformation. Inflow velocity $8 \mathrm{~m} / \mathrm{s}$, in propeller mode, was used to examine if the flexible blade can take airloads without a large negative bending. RPM range in propeller mode was found to be limited by the capacity of the motor. The tip mass position was selected as 0.25 to ensure CG is ahead of aerodynamic center. The full configuration of measurements is summarized in Table 5.

\subsection{Shape reconstruction and errors}

Fig. 10 demonstrates the process of blade reconstruction. LDS records the coordinate $z$ in the direction of height which is the distance from the blade surface on the measuring plane. RPM signal on motor rotation is obtained by optical sensor. Then, they are averaged in time domain as the corrections. After the correction of RPM, the polar coordinate based on mean rotational speed are transferred to Cartesian coordinate $x$ along spanwise direction and $y$ along chordwise direction to the measuring plane. Combining the coordinate $x, y$ and $z$, a polynomial surface fitting is performed to obtain the time-averaged shape of rotating blade. After the transformation of surface from measuring plane to reference plane, the real bending and torsion of rotating blade can be obtained. The coordinate $x$ and $y$ are calculated by local radius of each scanning, rotational speed and sampling time:

$$
\begin{aligned}
& x=r_{1} \cos \Omega t, \\
& y=r_{1} \sin \Omega t,
\end{aligned}
$$

where $t$ is the sampling time. In order to obtain the measurement precisions of coordinate $x$ and $y$, the Kline-McClintock method was used (Kline and McClintock, 1953). The precisions of coordinate $x$ and $y$ are defined by

$$
U_{\mathrm{x}}=\sqrt{\left(\frac{\partial x}{\partial r_{1}} U_{r_{1}}\right)^{2}+\left(\frac{\partial x}{\partial \Omega} U_{\Omega}\right)^{2}+\left(\frac{\partial x}{\partial t} U_{\mathrm{t}}\right)^{2}},
$$

Table 5

The configuration of measurements.

\begin{tabular}{lccc}
\hline Case & Rotor mode 1 (R1) & Rotor mode 2 (R2) & Propeller mode 1 (P1) \\
\hline RPM & 1100 & 1300 & 800 \\
Inflow velocity (m/s) & 0 & 0 & 8 \\
Collective pitch (deg) & 35 & 35 & 55 \\
Position factor of tip mass & 0.25 & 0.25 & 0.25 \\
\hline
\end{tabular}

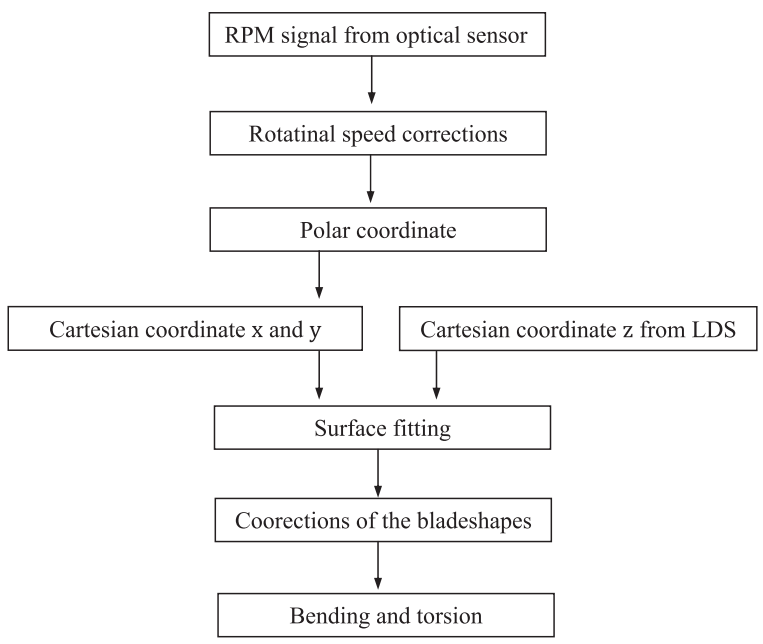

Fig. 10. Procedure of blade reconstruction. 


$$
U_{\mathrm{y}}=\sqrt{\left(\frac{\partial y}{\partial r_{1}} U_{r_{1}}\right)^{2}+\left(\frac{\partial y}{\partial \Omega} U_{\Omega}\right)^{2}+\left(\frac{\partial y}{\partial t} U_{\mathrm{t}}\right)^{2}}
$$

where $U_{r_{1}}$ is the uncertainty of the local radius, $U_{\Omega}$ is the uncertainty of the rotational speed and $U_{\mathrm{t}}$ is the uncertainty of the sampling time. Considering the RPM signal as the error source, the measurement precisions of coordinate $x$ and $y$ can be obtained based on the precisions of rotational speeds. In the experiments, the detecting range of LDS was from $-250 \mathrm{~mm}$ to $+500 \mathrm{~mm}$. According to the specification of LDS, the measuring accuracy of coordinate $z$ is $\pm 0.1 \%$ of Full Scale.

The multiple regression based on $\operatorname{3rd} \times 3$ rd polynomial equation to predict $z$ from $x$ and $y$ has the general form:

$$
z=p_{00}+p_{10} x+p_{01} y+p_{20} x^{2}+p_{11} x y+p_{02} y^{2}+p_{30} x^{3}+p_{21} x^{2} y+p_{12} x y^{2}+p_{03} y^{3}
$$

where $p_{i j}(i, j=0,1,2$ and 3 ) are the unknown coefficients. Writing Eq. (18) in the matrix form and leaving parameter vector $P$ at left side:

$$
P=M^{-1} Z
$$

where $Z$ is observation vector and $M$ is design matrix. With knowing the coordinate $x, y$ and $z$ of blade surface, parameter vector $P$ can be obtained, including residual vector. The coordinate defined to a reference plane can be obtained by transforming the coordinate on measuring plane, as shown in Fig. 11. All of the coordinate is transformed by a rotation matrix. Based on the coordinate $x^{\prime}, y^{\prime}$ and $z^{\prime}$ on reference plane, the exact bending and torsion of rotating blade can be obtained. In Fig. 12, an example is to show the shape reconstruction of a blade with complex shape. This is compared to the CAD model of the blade to get a good assessment of the reconstruction and the potential of this type of measurement. The tests on the blade were used to validate the measurement method and the post-processing algorithm.

\subsection{Fluid Structure Interaction model}

In order to compute the blade deformation under airloads and centrifugal loads, the Fluid Structure Interaction (FSI) model of the rotor combines the aerodynamic model - BEMT and structural analysis model - FBEAM (Mohd-Zawawi et al., 2013). The structural analysis is based upon the use of anisotropic beam finite element model to determine the blade deflections during operation. The FBEAM consists of an integrated set of programs, which perform at two-dimensional

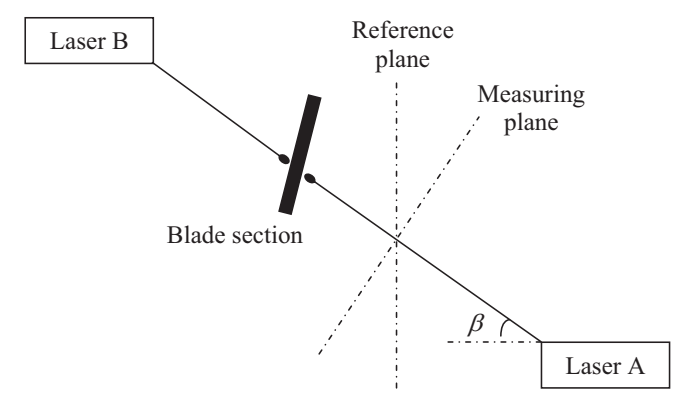

Fig. 11. Schematic of two coordinate systems.

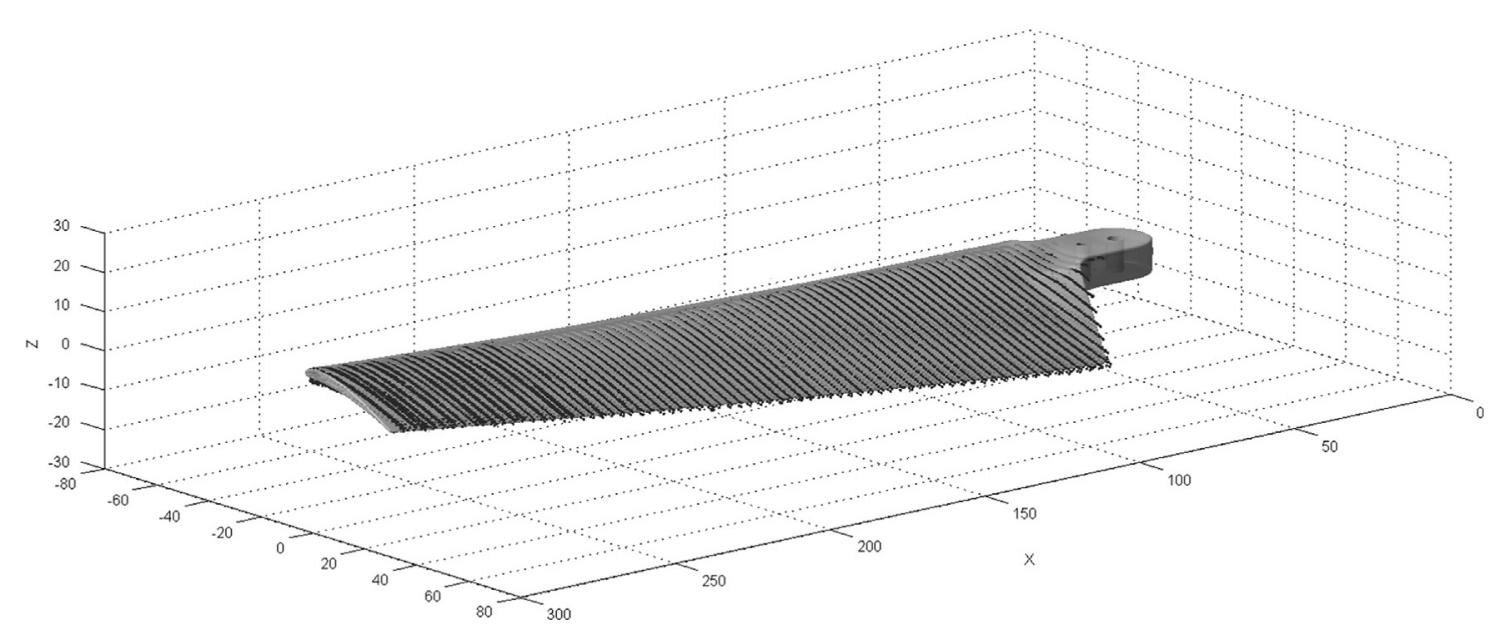

Fig. 12. An example of reconstruction for complex shaped blade. 
cross-sectional analysis of the blade, followed by a one-dimensional finite element of a proprotor blade. BECAS (Blasques and Lazarov, 2012), a cross-sectional analysis tool which is developed at Denmark Technical University (DTU) was incorporated in this structural analysis program. The motivation of employing BECAS in this two-step structural model is due to the fact that BECAS has ability to determine the cross section stiffness properties while accounting for all the geometrical and material induced couplings. These properties are also consequently utilized in the development of beam models to accurately predict the response of proprotor blades with complex geometries and made of advanced materials.

As the blade is modeled as a rotating, cantilever Timoshenko beam, featuring flapwise bending - lagwise bendingtorsion coupling, of arbitrary cross section and span, hence, expression of the elastic energy and kinetic energy of the beam element considering centrifugal force effect employed in the one-dimensional beam model is depicted in this section. By the consideration of coupling bend twist features, a wide range of structures can be then analysed. However, the blade with rectangular planform made up of laminated composite material is a focus in this study.

The detailed procedure of FSI model is shown in Fig. 13. The basic geometry (zero deflections) is assumed at the beginning. Then the structural model calculates the blade deformations under airloads and centrifugal loads. The deformed blade shape is used to update the aerodynamic model. The quasi-steady aerodynamic loads are computed for the update geometry, using the 2D aerodynamic theory based on BEMT. The aerodynamic loads are transferred to the beam nodes as concentrated forces. A new structural analysis is performed to calculate the deformed shape of the blade under the influence of aerodynamic and centrifugal loads. The variation of the blade twist angle along the blade is monitored for convergence. The interaction is repeated until equilibrium between deformation and loadings is achieved. In the structural model, the deformation is solved based on stiffness matrix. As a nonlinear consideration, the stiffness of each element changes during the deformation process, which is updated through the cross sectional analysis tool BECAS. After convergence the rotor performance characteristics are computed. The approach described above was applied to the constant chord untwisted 2-bladed system for small rotor made of laminate composite. The deflection results in two basic modes of deformation: bending and torsion.

\subsection{Results and discussion}

The measurement precisions of the coordinate $x$ and $y$ for blade reconstruction based on 3rd $\times$ 3rd polynomial surface are shown in Figs. 14 and 15. The coordinate $x$ of case P1 exhibits overall high precisions. This also happens to the precisions of coordinate $y$ of case P1. Since RPM signals are responsible for the precisions directly, the repeatability of rotational speed decreases with increasing RPM, as shown in Table 6. Fig. 16(a)-(c) demonstrates the residuals of each surface fitting. The values near blade root $(x=30 \mathrm{~mm})$ are high for all cases, since the surface fittings are mostly based on the LDS data acquired from blade surface instead of those at blade hub. In case P1, the high residuals are probably due to the dynamic vibration of rotating blade. Goodness-of-fit statistics in each case were used to evaluate the fitting quality, as shown in Table 7.

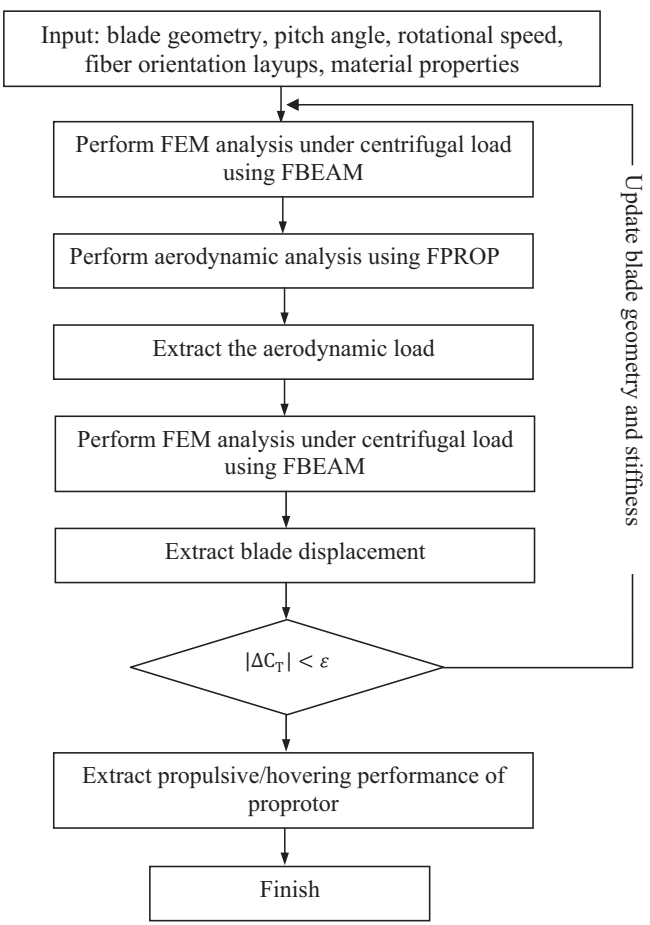

Fig. 13. Procedure of FSI analysis. 
(a)

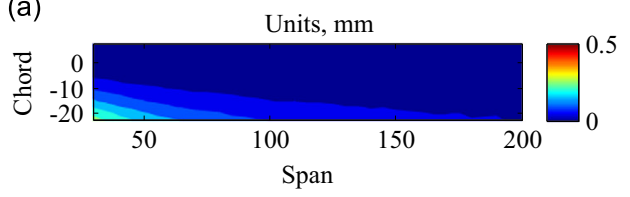

(b)

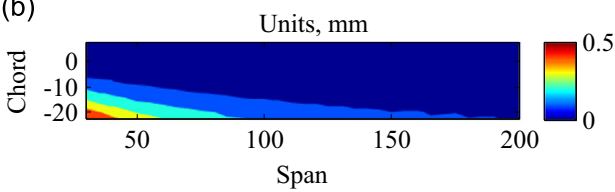

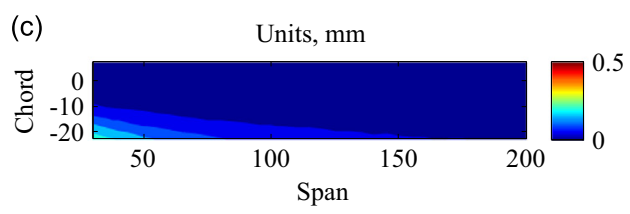

Fig. 14. Precisions of coordinate $x$ : (a) case R1; (b) case R2 and (c) case P1. (For interpretation of the references to color in this figure, the reader is referred to the web version of this article.)
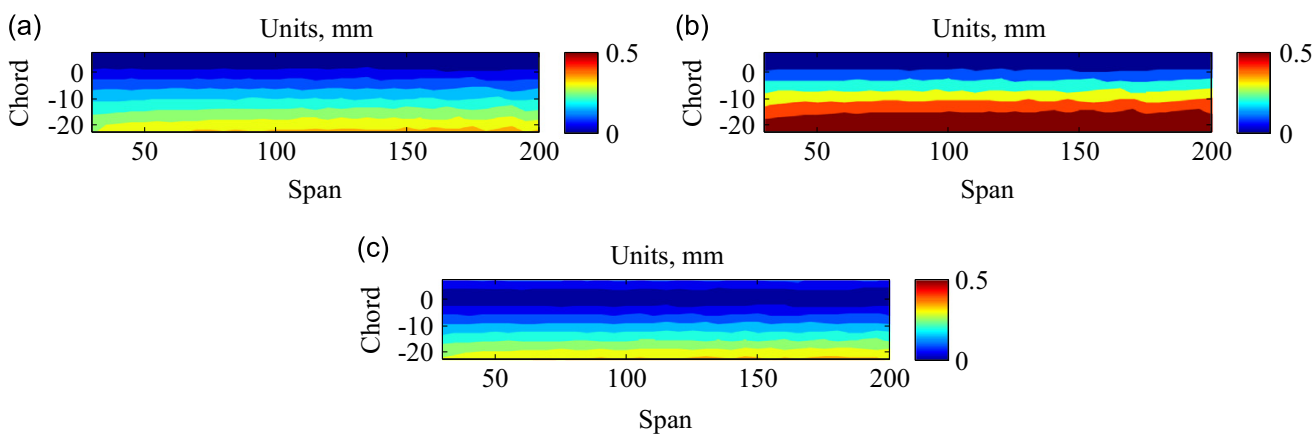

Fig. 15. Precisions of coordinate $y$ : (a) case R1; (b) case R2 and (c) case P1. (For interpretation of the references to color in this figure, the reader is referred to the web version of this article.)

Table 6

Measurement precisions of the rotational speeds.

\begin{tabular}{llll}
\hline Case & R1 & R2 & P1 \\
\hline Nominal RPM & 1100 & 1300 & 800 \\
Rotational speed (rad/s) & $122.08 \pm 1.64$ & $132.52 \pm 2.80$ & $85.99 \pm 1.33$ \\
\hline
\end{tabular}

(a)

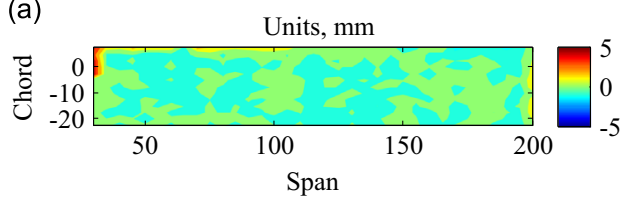

(b)

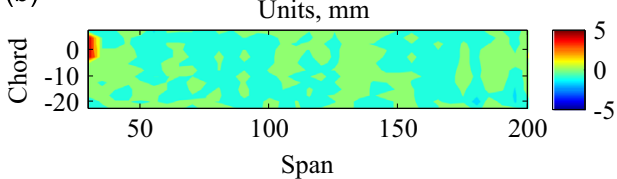

(c)

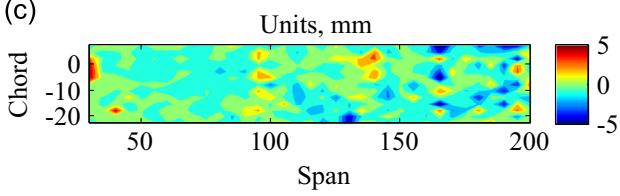

Fig. 16. Residuals of the fittings: (a) case R1; (b) case R2 and (c) case P1. (For interpretation of the references to color in this figure, the reader is referred to the web version of this article.)

The values of Sum of Squares due to Error (SSE) and Root Mean Squared Error (RMSE) are close to 0 that indicates the reliable fittings. By contrast, the values of the square of the correlation ( $R$-square) and Adjusted $R$-square according to the residual degrees of freedom (Adjusted $R$-square) are near to 1 indicating good fittings. Using the polynomial models with same order for cases R1, R2 and P1, the residuals of surface fitting for case P1 are relatively high, compared with R1 and R2 
Table 7

Goodness of fitting.

\begin{tabular}{llll}
\hline Case & $\mathrm{R} 1$ & $\mathrm{R} 2$ & $\mathrm{P} 1$ \\
\hline SSE & 0.0033 & 0.0038 & 0.0303 \\
$R$-square & 0.9967 & 0.9956 & 0.9888 \\
Adjusted $R$-square & 0.9967 & 0.9956 & 0.9888 \\
RMSE & $3.48 \mathrm{e}-4$ & $3.88 \mathrm{e}-4$ & $9.21 \mathrm{e}-4$ \\
\hline
\end{tabular}
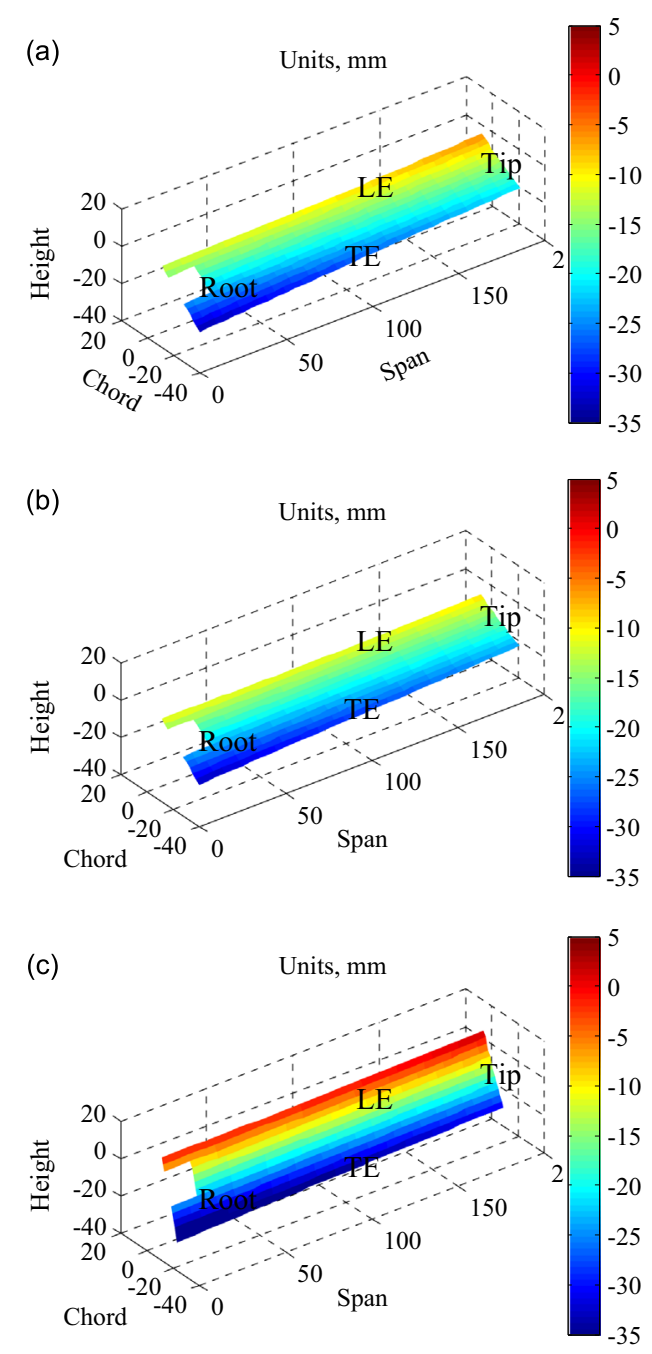

Fig. 17. 3D views of the reconstructed blades: (a) case R1; (b) case R2 and (c) case P1. (For interpretation of the references to color in this figure, the reader is referred to the web version of this article.)

cases (Fig. 16). This could indicate that the rotating blade has strong vibration behavior in propeller mode. The main reason to explain this phenomenon is that, at low RPM, tip mass induced torsion is small and therefore the propeller works in offdesign points and partially operates in stalled condition with separated flow induced vibration.

Fig. 17 shows the reconstructed blades rotating in rotor mode and propeller mode. The typical positions of blade were marked, such as blade root, blade tip, Leading Edge (LE) and Trailing Edge (TE). The data on blade hubs were eliminated and left blank areas. The blades rotate in the anti-clockwise direction along $Z$ axis. The color bar represents the height of blade. As it can be seen, all of three blades generated different levels of deformations. Fig. 18 gives the views of cross sections of the reconstructed blades. The slope ratio of blade tip is lower than the counterpart of blade root. It indicates the negative torsion occurred on the flexible blade. In cases R1, R2 and P1, the incidence angles at root are $32.5^{\circ}, 32.8^{\circ}$ and $51.7^{\circ}$, respectively. This agrees well with the initial collective pitches, which are $35^{\circ}, 35^{\circ}$ and $55^{\circ}$. Figs. 19,20 and 21 demonstrate the bending and 
(a)

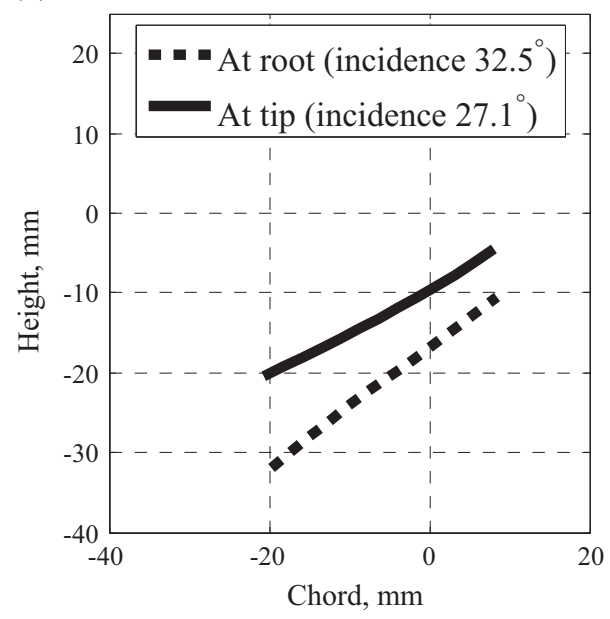

(b)

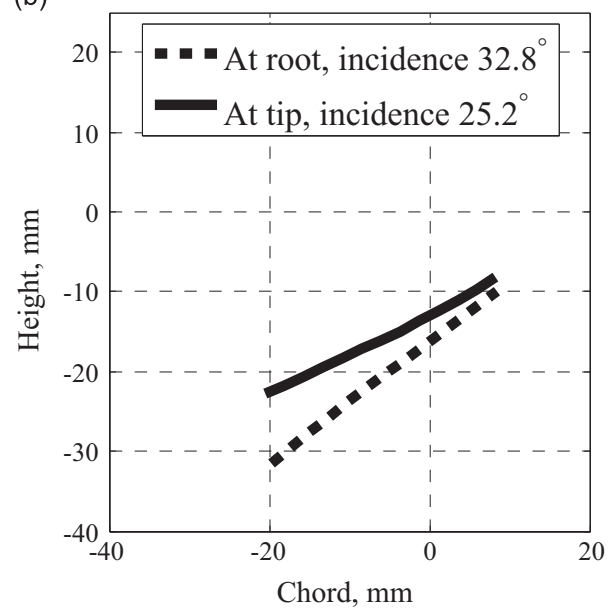

(c)

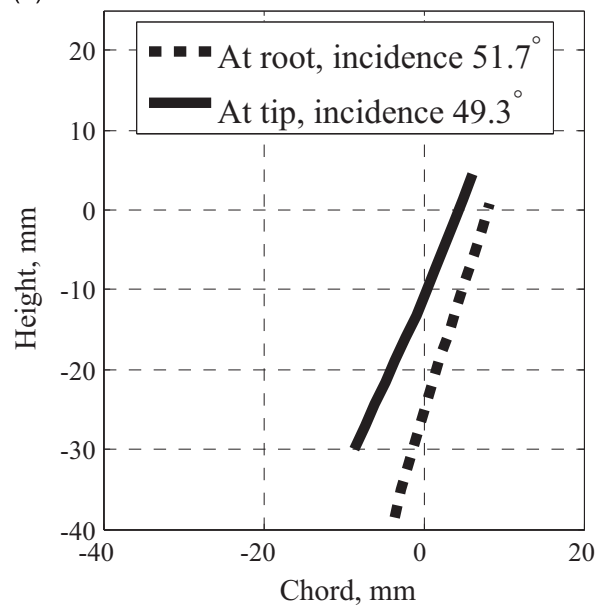

Fig. 18. Cross sections of the reconstructed blades: (a) case R1; (b) case R2 and (c) case P1.

torsion distributions of rotating blade in each case. The experimental results were plotted with 95\% confidence bounds of the fittings. In rotor mode, from case R1 (1100 RPM) to case R2 (1300 RPM), the tip bending from measurements decreased from $6.6 \mathrm{~mm}$ to $2.8 \mathrm{~mm}$ due to the centrifugal stiffening effect (Figs. 19(a) and 20(a)). By contrast, the experimental results show the tip torsion increases from $-5.4^{\circ}$ to $-7.6^{\circ}$ caused by a high nose-down pitching moment from increasing RPM (Figs. 19(b) and 20(b)). FSI model also reveals this phenomenon between cases R1 and R2. According to the results of 
(a)

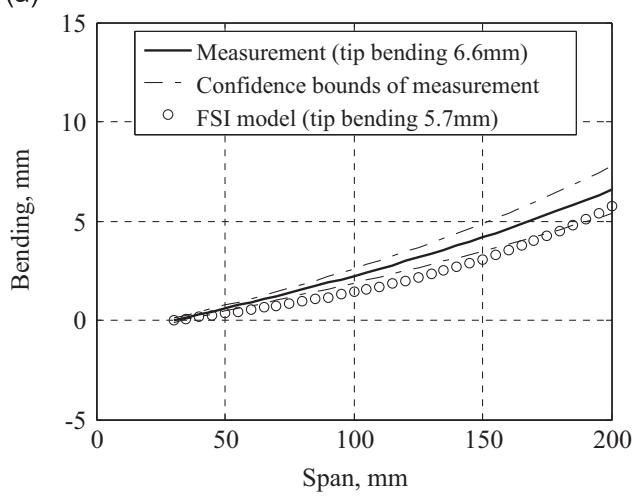

(b)

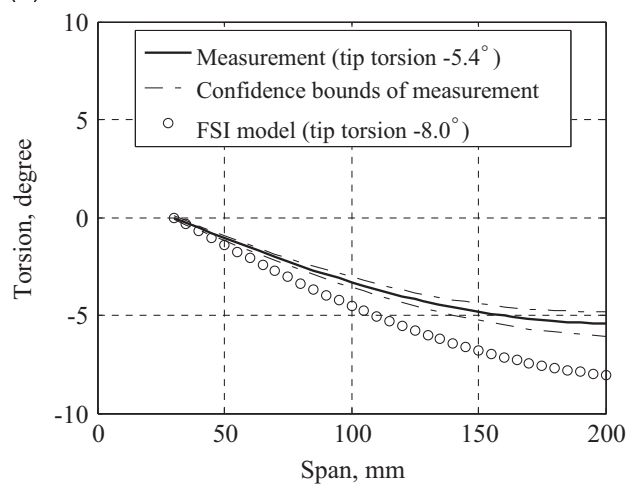

Fig. 19. Deformation of case R1: (a) bending and (b) torsion.

experiments and FSI model, in case P1 (Fig. 21(a)), even RPM is only 800 (low RPM has no strong stiffening effect), the bending still is lower than case R1 (Fig. 19(a)). This is because of the inflow airloads acting on the blade. The tip torsion is only $-2.4^{\circ}$ since there was no adequate nose-down pitching moment in this case (Fig. 21(b)). The large bounds of experimental results in case P1 are probably from the unsteady effect of airloads. As discussed above, the low RPM cannot produce the desired optimum twist for propeller mode. Hence, the propeller works with some stalled airfoil sections and the separated flow could induce the blade vibration. The results show CFIT blade is capable of producing the variable and beneficial torsion for two flight modes. However, the desired torsion did not reach according to the optimum twist rates which are $-10^{\circ}$ for hover and $-30^{\circ}$ for forward flight respectively, especially in propeller mode. FSI model is capable of providing the same trends with the measurements. It was found that FSI model always over predicts the torsion for all of the cases. Actually, at blade tip, an aluminum tube was enclosed in the carbon fiber/epoxy assembling with the glass/epoxy blade (Fig. 7). The additional part should increase the local torsional stiffness of glass fiber/epoxy blade. Since it is difficult to find the torsional stiffness of the flexible proprotor blade theoretically, we assume that the blade in FSI model is purely made up of glass fiber. The torsional stiffness of the flexible blade is expected to be obtained experimentally. Besides, the difference could be from the aerodynamic model. For example, if the operation condition of rotating blade is at off-design points, the accuracy will decrease due to the non-streamlined flow. Furthermore, even accurate 2D airfoils characteristics are used, but it is not capable of considering the 3D rotating effect. And the rotating effect could change the stall behavior of the sectional airfoil.

\section{Conclusion}

An aerodynamic model was developed to study the twist effect for hover and forward flight based on BEMT method. The results show low twist is beneficial for hover while high twist is helpful for forward flight in terms of aerodynamic efficiency. A CFIT flexible blade was proposed for small-scale rotor to increase the overall efficiency in aerodynamics. The tip mass was carefully selected to improve the stability of rotating flexible blade and provide nose-down pitching moment for a negative torsion. CLT model was used to examine the CFIT concept. The result indicates CFIT blade has the possibility to produce the desired torsion. A LDS rig and corresponding post-processing method were developed to reconstruct the rotating blade. Experimental results show that, in rotor mode, with increasing RPM, the bending decreases due to the centrifugal stiffening effect. By contrast, the increasing centrifugal nose-down moment increases the blade torsion. In hover, the maximum value of blade tip torsion is $-7.6^{\circ}$, which is close to the desired value of $-10^{\circ}$. In propeller mode, the flexible 
(a)

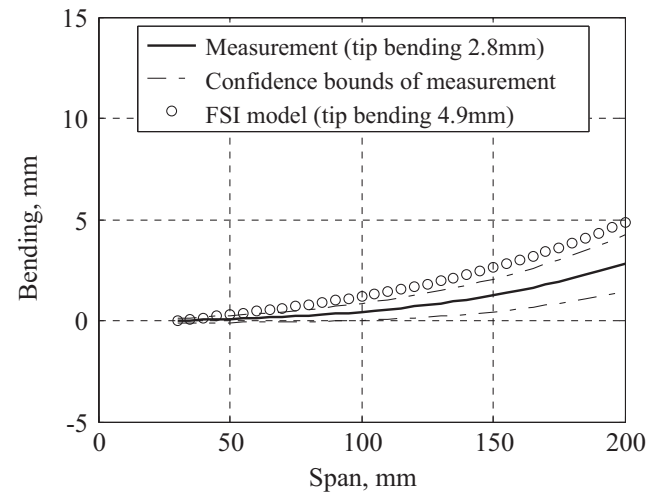

(b)

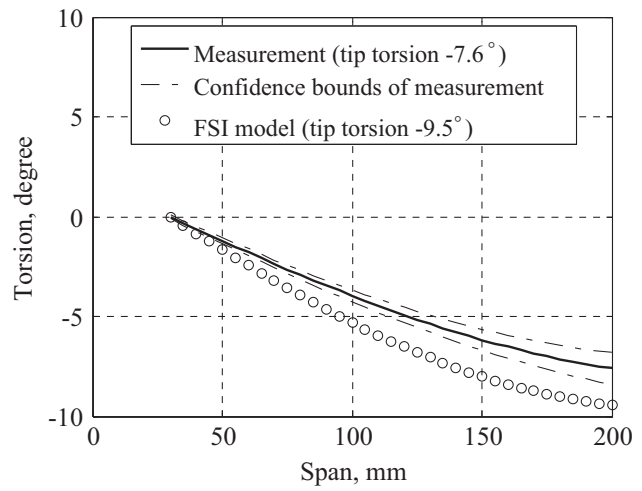

Fig. 20. Deformation of case R2: (a) bending and (b) torsion.

(a)

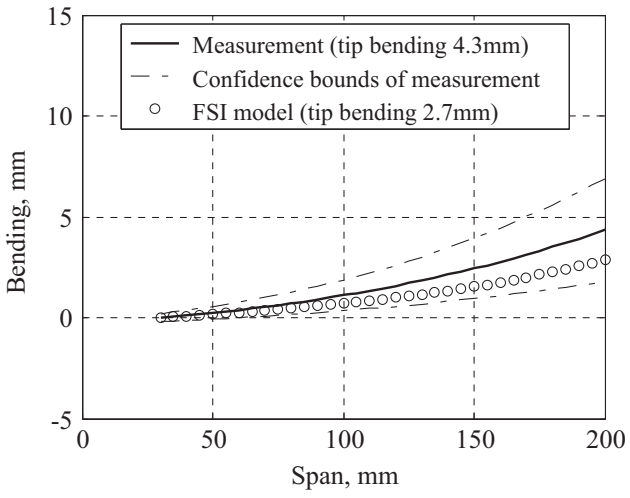

(b)

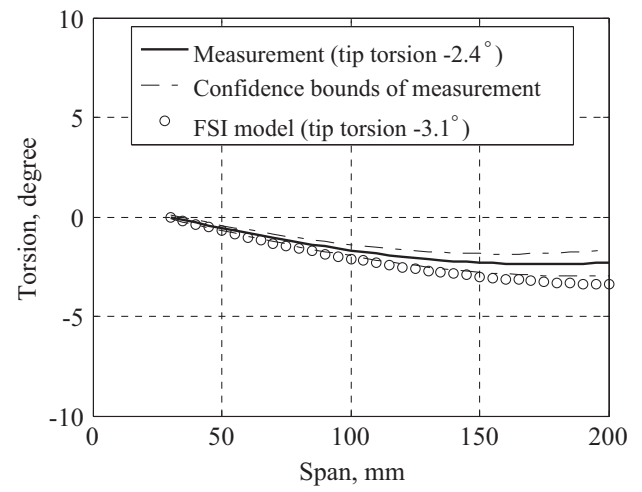

Fig. 21. Deformation of case P1: (a) bending and (b) torsion. 
blade can take airloads without large negative bending. However, due to the limitation of RPM, the tip torsion is only $-2.4^{\circ}$ for forward flight. The deformation did not achieve the desired tip torsion of $-30^{\circ}$. FSI model gives the same trends with experimental results. The LDS rig is capable of measuring the deformation even for a small-scale blade. The CFIT blade can produce variable and negative torsion for two flight modes. In order to achieve high tip torsion in forward flight, the deformation tests at a high RPM is expected in propeller mode.

\section{Acknowledgments}

The authors thank Rémy Chanton and Xavier Foulquier for the advice and assistance in experiments. The research is supported by China Scholarship Council (CSC) and Ministry of Higher Education, Malaysia (MOHE).

\section{References}

Adkins, C.N., 1990. Design of optimum propellers. Journal of Propulsion and Power 10, 676-682.

Bauchau, O.A., Loewy, R.G., Bryan, P.S., 1983. An approach to ideal twist distribution in tilt rotor VSTOL blade designs. In: Proceedings of the 39th Annual Forum of the American Helicopter Society. St. Louis, MO.

Blasques, J.P., Lazarov, B., 2012. Becas v2. Technical Report-R 1785

Bohorquez, F., 2007. Rotor Hover Performance and System Design of an Efficient Coaxial Rotary Wing Micro Air Vehicle (Ph.D. thesis). University of Maryland.

Daniel, I.M., Ishai, O., 1994. Engineering Mechanics of Composite Materials. Oxford University Press.

Drela, M., 1989. An analysis and design system for low Reynolds number airfoil, Low Reynolds Number AerodynamicsSpringer-Verlag, New York1-12.

Fleming, G.A., Gorton, S., 1998. Measurement of rotorcraft blade deformation using projection Moire interferometry. In: Proceedings of the Third International Conference on Vibration Measurements by Laser Techniques: Advances and Applications. SPIE - The International Society for Optical Engineering, Ancona, Italy, pp. 514-527.

Fleming, G.A., Soto, H.L., South, B.W., 2002. Projection Moiré interferometry for rotorcraft application: deformation measurements of active twist rotor blades. In: Proceedings of the 58th Annual Forum of the American Helicopter Society. Montréal, Canada.

Hein, B.J., Chopra, I., 2005. Hover performance of a micro air vehicle: rotors at low Reynolds number. In: Proceedings of the International Specialists Meeting Unmanned Rotorcraft: Design, Control and Testing. Chandler, AZ, USA.

Kline, S.J., McClintock, F.A., 1953. Describing uncertainties in single-sample experiments. Mechanical Engineering 75, 3-8.

Lake, R.C., Nixon, M.W., Wilbur, M.L., Singleton, J.D., Mirick, P.H., 1994. Demonstration of an elastically coupled twist control concept for tilt rotor blade application. AIAA Journal 32 (7), 1549-1551.

Lawson, M.S., Sirohi, J., 2011. Measurement of deformation of rotating blades using digital image correlation. In: Proceedings of the 52nd AIAA/ASME/ASCE/ AHS/ASC Structures, Structural Dynamics and Materials Conference, AIAA 2011-1876. Denver, Colorado, pp. 1-15.

Leishman, J.G., 2006. Principles of helicopter aerodynamics, Cambridge Aerospace Series2nd edition.

McVeigh, M.A., Rosenstein, H.J., 1983. Aerodynamic design of the XV-15 advanced composite tilt rotor blade. In: Proceedings of the 39th Annual Forum of the American Helicopter Society, pp. 72-80.

Mohd-Zawawi, F., Prothin, S., Lv, P., Benard, E., Moschetta, J.M., Morlier, J., 2013. Study of a flexible UAV proprotor. In: Proceedings of the 48th International Symposium of Applied Aerodynamics, vol. 30. Saint-Louis, pp. 1-10.

Nixon, M.W., 1987. Extension-twist coupling of composite circular tubes with application to tiltrotor blade design. In: Proceedings of the AIAA/ASME/ASCE/ AHS/ASC Structures, 28th Structural Dynamics and Materials Conference. Monterey, CA, pp. 295-303.

Nixon, M.W., 1988. Improvements to Tilt-Rotor Performance Through Passive Blade Twist Control. NASA Technical Memorandum 100583.

Nixon, M.W., Piatak, D.J., Corso, L.M., Popelka, D.A., 2000. Aeroelastic tailoring for stability augmentation and performance enhancements of tiltrotor aircraft. Journal of the American Helicopter Society 45 (4), 270-279.

Olson, L., Abrego, A., Barrows, D., Burner, A., 2010. Blade deflection measurements of a full-scale UH-60A rotor system. In: Proceedings of the American Helicopter Society Aeromechanics Specialist Conference. San Francisco, California.

Rajpal, S., 2009. Optical Methods of Measurement: Whole-Field Techniques, second edition Francis and Taylor/CRC Press.

Schmidt, T., Tyson, J., 2003. Full-field dynamic displacement and strain measurement using advanced 3D image correlation photogrammetry: Part 1. Experimental Techniques 27 (3), 47-50.

Shkarayev, S., Moschetta, J.M., Bataille, B., 2008. Aerodynamic design of micro air vehicles for vertical flight. Journal of Aircraft 45, 1715-1724.

Sicard, J., 2011. Investigation of an Extremely Flexible Stowable Rotor for Micro-helicopters (Master's thesis). University of Texas at Austin.

Smedresman, A., Yeo, D., Shyy, W., 2011. Design, fabrication, analysis, and testing of a micro air vehicle propeller. In: Proceedings of the 29th AIAA Applied Aerodynamics Conference, AIAA 2011-3817. Honolulu, Hawaii, pp. 1-16.

Winston, M.M., 1968. An Investigation of Extremely Flexible Lifting Rotors. NASA Technical Note, NASA TN D-4465, pp. 1-27. 